\title{
The FBK Participation in the WMT15 Automatic Post-editing Shared Task
}

\author{
Rajen Chatterjee \\ Fondazione Bruno Kessler \\ chatterjee@flok. eu
}

\author{
Marco Turchi \\ Fondazione Bruno Kessler \\ turchiefbk.eu
}

\author{
Matteo Negri \\ Fondazione Bruno Kessler \\ negridfbk.eu
}

\begin{abstract}
In this paper, we describe the "FBK EnglishSpanish Automatic Post-editing (APE)" systems submitted to the APE shared task at the WMT 2015. We explore the most widely used statistical APE technique (monolingual) and its most significant variant (context-aware). In this exploration, we introduce some novel task-specific dense features through which we observe improvements over the default setup of these approaches. We show these features are useful to prune the phrase table in order to remove unreliable rules and help the decoder to select useful translation options during decoding. Our primary APE system submitted at this shared task performs significantly better than the standard APE baseline.
\end{abstract}

\section{Introduction}

Over the last decade a lot of research has been carried out to mimic the human post-editing process in the field of Automatic Post-Editing (APE). The objective of APE is to learn how to correct machine translation (MT) errors leveraging the human post-editing feedback. The variety of data generated by human feedback, in terms of post editing, possess an unprecedented wealth of knowledge about the dynamics (practical and cognitive) of the translation process. APE leverages the potential of this knowledge to improve MT quality. The problem is appealing for several reasons. On one side, as shown by Parton et al. (2012), APE systems can improve MT output by exploiting information unavailable to the decoder, or by performing deeper text analysis that is too expensive at the decoding stage. On the other side, APE represents the only way to rectify errors present in the "black-box" scenario where the MT system is unknown or its internal decoding information is not available.
The goal of the APE task is to challenge the research groups to improve the MT output quality by the use of a dataset consisting of triplets of sentences (source, MT output, human post-edition). We are facing the "MT-as-Black-box" scenario, so neither we have access to the MT engine nor do we have any decoding trace. The data for this pilot task belongs to generic news domain which reflects data sparseness, and the post-edition of the MT output is obtained through crowdsourcing which makes it vulnerable to noise thus making this task even more challenging.

To begin with, $\S 2$ discusses the statistical APE methods used to implement the APE systems. $§ 3$ describes the data set available for this shared task, and provides detail of the experimental setup. $\S 4$ is our major contribution which discusses the FBK-APE pipeline and shows that incorporation of task-specific dense features can be useful to enhance APE systems. Our final submitted system is reported in $\S 5$ followed by conclusion in $\S 6$.

\section{Statistical APE Methods}

In this paper we examine the most widely used statistical phrase-based post-editing strategy proposed by Simard et al. (2007) and its most significant variant proposed by Béchara et al. (2011). We describe the two methods and there pros and cons in the following subsections.

\subsection{APE-1 (Simard et al., 2007)}

In this approach APE systems are trained in the same way as the statistical machine translation (SMT) system. But, as contrast to SMT which makes use of the source and target language parallel corpus, APE uses the MT output and its corresponding human post-edited data in the form of parallel corpus. One of the most important missing concepts in this "monolingual translation" is the inclusion of source information, which has been incorporated in the next approach. 


\subsection{APE-2 (Béchara et al., 2011)}

This technique is the most significant variant of (Simard et al., 2007), where they come up with a new data representation to include the source information along with the MT output on the source side of the parallel corpus. For each MT word $f^{\prime}$, the corresponding source word (or phrase) $e$ is identified through word alignment and used to obtain a joint representation $f^{\prime} \# e$. This results in a new intermediate language $F^{\prime} \# E$ that represents the new source side of the parallel data used to train the statistical APE system. This "context-aware" variant seems to be more precise but faces two potential problems. First, preserving the source context comes at the cost of a larger vocabulary size and, consequently, higher data sparseness that will eventually reduce the reliability of the translation rules being learned. Second, the joint representation $f^{\prime} \# e$ may be infected by the word alignment errors which may mislead the learning of translation option.

Recently, Chatterjee et al. (2015) showed a fair systematic comparison of these two approaches over multiple language pairs and revealed that inclusion of source information in the form of context-aware variant is useful to improve translation quality over standard monolingual translation approach. They also showed that using monolingual translation alignment to build context-aware APE helps to mitigate the sparsity issue at the level of word alignment and for this reasons, we use this configuration to implement APE-2 method.

\section{Data set and Experimental setup}

Data: In this shared task we are provided with a tri-parallel corpus consisting of source (src), MT output (mt), and human post-edits (pe). While APE-1 uses only the last two elements of the triplet, all of them are used in the context-aware APE-2. To obtain joint representation $\left(f^{\prime} \# e\right)$ in APE-2, word alignment model is trained on $s r c-m t$ parallel corpus of the training data. The training set consist of $\sim 11 \mathrm{~K}$ triplets, we divide the development set into dev and test set consisting of 500 triplets each. Our evaluation is based on the performance achieved on this test set. We tokenize the data set using the tokenizer available in the MOSES(Koehn et al., 2007) toolkit. Training and evaluation of our APE systems are performed on the true-case data.

Experiment Settings: To develop the APE systems we use the phrase-based statistical machine translation toolkit MOSES(Koehn et al., 2007). For all the experiments mentioned in this paper we use "grow-diag-final-and" as alignment heuristic and "msd-bidirectional-fe" heuristic for reordering model. MGIZA++ (Gao and Vogel, 2008) is used for word alignment. The APE systems are tuned to optimize TER(Snover et al., 2006) with MERT(Och, 2003).

We follow an incremental strategy to develop the APE systems, at each stage of the APE pipeline we find the best configuration of a component and then proceed to explore the next component. Our APE pipeline consist of various stages like language model selection, phrase table pruning, and feature designing as discussed in the following sections.

Evaluation Metric: We select TER (Snover et al., 2006) as our evaluation metric because it mimics the human post-editing effort by measuring the edit operation needed to translate the MT output into its human-revised version.

Apart from TER as an evaluation metric we also compute number of sentences being modified $^{1}$ in the test set and then compute the precision as follow:

$$
\text { Precision }=\frac{\text { Numberof SentencesImproved }}{\text { Numberof SentencesModified }}
$$

Baseline: Our baseline is the MT output as$i s$. To evaluate, we use the corresponding human post-edited corpus which gives us 23.10 TER score.

\section{APE Pipeline}

In this section we describe various components that we explore at each stage of the pipeline. At each stage, we study the effect of several configuration of each component on both the APE methods (APE-1 and APE-2)

\subsection{Language Model Selection (APE-LM)}

We use various data set to train multiple language models to see which of them have high impact on the translation quality. All the LMs are trained us-

\footnotetext{
${ }^{1}$ For each sentence in the test set, if the TER score of APE system is different than the baseline then we consider it as a modified sentence
} 
ing IRSTLM toolkit (Federico et al., 2008) having order of 5 gram with kneser-ney smoothing. The data set varies in quality and quantity as described below:

- LM 1 contains only the training data $(\sim 11 \mathrm{~K})$ provided in this shared task. Although the data set contains few sentences to train a language model compared to the data used in MT, it is quite reliable because it is sampled from the same distribution of the test set.

- LM 2 consists of News Commentary having $\sim 200 \mathrm{~K}$ sentences, downloaded from WMT 2013 translation task. ${ }^{2}$ This corpus belongs to the same domain of the APE data, but it is created under different conditions (i.e. involving professional translators and translating from scratch the source sentence) making it significantly different from the data used to build LM1.

- LM 3 (Big data) contains News Crawl data from 2007-2012 contributing to $\sim 13 \mathrm{M}$ sentences, downloaded from WMT 2013 translation task ${ }^{2}$. This data set has huge amount of news crawled from the Web and covering several topics.

- LM1+LM2+LM3: All the previous language models are simultaneously used by the APE systems. A log-linear weight is assigned to each language model during the tuning stage.

\begin{tabular}{|l|c|c|}
\hline & APE-1 & APE-2 \\
\hline LM1 & $\mathbf{2 3 . 9 5}$ & $\mathbf{2 4 . 5 9}$ \\
\hline LM2 & 23.96 & 24.62 \\
\hline LM3 & 24.06 & 24.66 \\
\hline LM1+LM2+LM3 & 24.05 & 24.69 \\
\hline
\end{tabular}

Table 1: Performance (TER score) of the APE systems using various LMs

Results of both the APE systems are shown in Table 1. We notice that the performance of the APE systems do not show much variation for different LMs. This can come from the fact that the news commentary and new crawl data might not resemble well the shared task data. For this reason, the in-domain LM1 is selected and used in the next stages.

\footnotetext{
${ }^{2}$ http://www.statmt.org/wmt13/translation-task.html
}

\subsection{Pruning Strategy (APE-LM1-Prun)}

To remove unreliable translation rules generated from the data obtained through crowd-sourcing, pruning strategies are investigated. First, we test the classic pruning technique by Johnson et al. (2007) which is based on the significance testing of phrase pair co-occurrence in the parallel corpus. According to our experiments, this technique is too aggressive when applied on limited amounts of sparse data. Nearly $5 \%$ of the phrase table is retained after pruning with mostly self-rules (translation options that contain same source and target phrase).

For this reason we develop a novel feature for pruning which measures the usefulness of a translation option present in the phrase table. For each translation option in the phrase table, all the parallel sentences are retrieved from the training set such that the source phrase of the translation option is present in the source sentence of the parallel corpus. We then substitute the target phrase of the translation option in the source sentence of the parallel corpus and then compute the TER score wrt. the corresponding target sentence. If TER increases then we increment the neg-count by 1 , and if TER decreases we increment the pos-count by 1 . Finally, we compute the neg-impact and the pos-impact as follows:

$$
\begin{aligned}
\text { neg-impact } & =\frac{\text { neg-count }}{\text { NumberofRetrievedSentences }} \\
\text { pos-impact } & =\frac{\text { pount }}{\text { NumberofRetrievedSentences }}
\end{aligned}
$$

Once these ratios are computed for all translation options, we filter the phrase table by thresholding on the neg-impact to remove rules which are not useful (higher the neg-impact less useful it is). All translation options greater than or equal to the threshold value are filtered out. We apply this pruning strategy for both the APE methods over various threshold values.

Table 2 and Table 3 show the performance after pruning the APE-1-LM1 and APE-2-LM1 systems respectively. In Table 2, we observe that TER score for various threshold values are very close to each other, so in order to select the best threshold value we base our decision on precision. So for APE-1, we select the threshold value of 0.4 which shows the highest precision, namely APE1-LM1-Prun0.4. For APE-2, it is evident from the result in Table 3 that the threshold value of 0.2 


\begin{tabular}{|c|c|c|c|}
\hline Threshold & TER & $\begin{array}{c}\text { Number of } \\
\text { sentences } \\
\text { modified }\end{array}$ & Precision \\
\hline 0.8 & 23.90 & 88 & 0.12 \\
\hline 0.6 & 23.91 & 90 & 0.13 \\
\hline $\mathbf{0 . 4}$ & 23.98 & 94 & $\mathbf{0 . 1 5}$ \\
\hline 0.2 & 23.77 & 70 & 0.12 \\
\hline
\end{tabular}

Table 2: Performance (TER score) of the APE-1LM1 after pruning at various threshold values

\begin{tabular}{|c|c|c|c|}
\hline Threshold & TER & $\begin{array}{c}\text { Number of } \\
\text { sentences } \\
\text { modified }\end{array}$ & Precision \\
\hline 0.8 & 24.29 & 130 & 0.20 \\
\hline 0.6 & 23.99 & 103 & 0.18 \\
\hline 0.4 & 23.66 & 70 & 0.18 \\
\hline $\mathbf{0 . 2}$ & 23.46 & 50 & $\mathbf{0 . 2 2}$ \\
\hline
\end{tabular}

Table 3: Performance (TER score) of the APE-2LM1 after pruning at various threshold values

proves to be the best in terms of TER score (reduction by 1.13 point) as well as in terms of precision (APE-2-LM1-Prun0.2). These results suggest that our pruning technique has a larger impact on the APE-2 method compared to APE- 1 . This is motivated by the fact that the context-aware approach is affected by the data sparseness problem resulting in a large number of unreliable translation options that can be removed from the phrase table.

\subsection{New Dense Features Design}

The final stage of our APE pipeline is the feature design. When a translation system is trained using Moses, it generates translation model consisting of default dense features like phrase translation probability (direct and indirect) and lexical translation probability (direct and indirect). In the task of Automatic Post-editing where we have the source and target phrases in the same language, we can leverage this information to provide the decoder with some useful insights. In the light of this direction we design four task-specific dense features to raise the "awareness" of the decoder.

\section{- Similarity $(f 1)$ :}

This feature $(f 1)$ is quite similar to the one proposed in (Grundkiewicz and JunczysDowmunt, 2014) which measures the similarity between the source and target phrase of the translation options. The score for $f 1$ is computed as follows:

$$
f 1_{\text {score }}=e^{1-\operatorname{ter}(s, t)}
$$

where ter measures the number of edit operations required to translate the source phrase $s$ to the target phrase $t$ and it is computed using TER(Snover et al., 2006).

- Reliability ( $f 2.1$ and $f 2.2)$ :

We allow the model to learn the reliability of the translation option by providing it with the statistics of the quality (in terms of HTER) of the parallel sentences used to learn that particular translation option. Better the quality, higher the likelihood to learn reliable rules. For each translation option in the phrase table, all the parallel sentence pairs from the training data containing the source phrase in the machine translated sentence of the pair and target phrase in the post-edited sentence are retrieved along with their HTER score. These scores are then used to compute the following two features:

Median ( $f 2.1$ ): The median of the HTER values of all the retrieved pairs.

Standard Deviation $(f 2.2)$ : The standard deviation of the HTER values of all the retrieved pairs.

- Usefulness $(f 3)$ : As discussed in Section 4.2 we use pos-impact as a feature to measure the positive impact of a translation option over the training set. Higher the positive impact, higher is its usefulness.

We study the impact of individual features when applied one at a time and when used all together.

\begin{tabular}{|c|c|c|c|}
\hline Features & TER & $\begin{array}{c}\text { Number of } \\
\text { sentences } \\
\text { modified }\end{array}$ & Precision \\
\hline$f 1$ & 23.87 & 81 & 0.16 \\
\hline $\boldsymbol{f 2 . 1 , \boldsymbol { f 2 . 2 }}$ & 23.92 & 94 & $\mathbf{0 . 1 9}$ \\
\hline$f 3$ & 23.88 & 82 & 0.14 \\
\hline$f 1, f 2.1$, & 23.97 & 85 & 0.12 \\
$f 2.2, f 3$ & & & \\
\hline
\end{tabular}

Table 4: Performance (TER score) of the APE-1LM1-Prun0.4 for different features

Table 4 and Table 5 show the performance of various features for APE-1-LM1-prun0.4 and 


\begin{tabular}{|c|c|c|c|}
\hline Features & TER & $\begin{array}{c}\text { Number of } \\
\text { sentences } \\
\text { modified }\end{array}$ & Precision \\
\hline $\boldsymbol{f 1}$ & 23.50 & 52 & $\mathbf{0 . 2 7}$ \\
\hline$f 2.1, f 2.2$ & 23.50 & 53 & 0.20 \\
\hline$f 3.1$ & 23.52 & 59 & 0.22 \\
\hline$f 1, f 2.1$, & 23.52 & 54 & 0.19 \\
$f 2.2, f 3.1$ & & & \\
\hline
\end{tabular}

Table 5: Performance (TER score) of the APE-2LM1-Prun0. 2 for different features

APE-2-LM1-Prun0.2 systems respectively. We observe, on this data set, that the use of these features retains the APE performance in terms of TER score but slight improvement is observed in terms of precision over both the APE systems, which indicate its contribution to improve the translation quality.

\section{Final Submitted Systems}

Our primary system is the best system in Table 5 i.e. APE-2-LM1-Prun0.2-f1 and contrastive system is the best system in Table 4 i.e. APE-1-LM1Prun0.4-f2.1-f2.2. According to the shared task evaluation report the scores of our submitted systems are shown in Table 6

\begin{tabular}{|l|c|c|}
\hline Systems & $\begin{array}{c}\text { Case } \\
\text { Sensitive }\end{array}$ & $\begin{array}{c}\text { Case In- } \\
\text { sensitive }\end{array}$ \\
\hline Baseline (MT) & 22.91 & 22.22 \\
\hline $\begin{array}{l}\text { APE Baseline } \\
\text { (Simard et al., 2007) }\end{array}$ & 23.83 & 23.13 \\
\hline Primary & 23.22 & 22.55 \\
\hline Contrastive & 23.64 & 22.94 \\
\hline
\end{tabular}

Table 6: APE shared task evaluation score (TER)

Although we could not beat the Baseline (MT), but we see a clear improvement over APE baseline (Simard et al., 2007) by the inclusion of our novel features and the use of the pruning strategy.

\section{Conclusion}

The APE shared task was challenging in many terms (black-box MT, generic news domain data, crowdsourced post-editions). Though we were unable to beat the MT baseline but we gained some positive experience through this shared task. First, our primary APE system performed significantly better ( 0.61 TER reduction) over the standard APE baseline (Simard et al., 2007) as reported in Table 6. Second, our novel dense feature (neg-impact) used to prune phrase table shows significant improvement in the context-aware APE performance. Third, other task-specific dense features which measure similarity and reliability of the translation options help to improve the precision of our APE systems. To encourage the use of our features we have publicly released the scripts at https: //bitbucket.org/turchmo/apeatfbk/ src/master/papers/WMT2015/APE_ 2015_System_Scripts.zip.

\section{Acknowledgements}

This work has been partially supported by the ECfunded H2020 project QT21 (grant agreement no. 645452).

\section{References}

Hanna Béchara, Yanjun Ma, and Josef van Genabith. 2011. Statistical post-editing for a statistical mt system. In MT Summit, volume 13, pages 308-315.

Rajen Chatterjee, Marion Weller, Matteo Negri, and Marco Turchi. 2015. Exploring the Planet of the APEs: a Comparative Study of State-of-the-art Methods for MT Automatic Post-Editing. In Proceedings of the 53rd Annual Meeting of the Association for Computational Linguistics), Beijing, China.

Marcello Federico, Nicola Bertoldi, and Mauro Cettolo. 2008. Irstlm: an open source toolkit for handling large scale language models. In Interspeech, pages 1618-1621.

Qin Gao and Stephan Vogel. 2008. Parallel implementations of word alignment tool. In Software Engineering, Testing, and Quality Assurance for Natural Language Processing, pages 49-57. Association for Computational Linguistics.

Roman Grundkiewicz and Marcin Junczys-Dowmunt. 2014. The amu system in the conll-2014 shared task: Grammatical error correction by data-intensive and feature-rich statistical machine translation. CoNLL-2014, page 25.

John Howard Johnson, Joel Martin, George Foster, and Roland Kuhn. 2007. Improving translation quality by discarding most of the phrasetable.

Philipp Koehn, Hieu Hoang, Alexandra Birch, Chris Callison-Burch, Marcello Federico, Nicola Bertoldi, Brooke Cowan, Wade Shen, Christine Moran, Richard Zens, et al. 2007. Moses: Open source toolkit for statistical machine translation. In Proceedings of the 45th annual meeting of the ACL on 
interactive poster and demonstration sessions, pages 177-180. Association for Computational Linguistics.

Franz Josef Och. 2003. Minimum error rate training in statistical machine translation. In Proceedings of the 41st Annual Meeting on Association for Computational Linguistics-Volume 1, pages 160-167. Association for Computational Linguistics.

Kristen Parton, Nizar Habash, Kathleen McKeown, Gonzalo Iglesias, and Adrià de Gispert. 2012. Can automatic post-editing make $\mathrm{mt}$ more meaningful. Proceeding EAMT, 12:111-118.

Michel Simard, Cyril Goutte, and Pierre Isabelle. 2007. Statistical Phrase-Based Post-Editing. In Proceedings of the Annual Conference of the North American Chapter of the Association for Computational Linguistics (NAACL HLT), pages 508-515, Rochester, New York.

Matthew Snover, Bonnie Dorr, Richard Schwartz, Linnea Micciulla, and John Makhoul. 2006. A study of translation edit rate with targeted human annotation. In Proceedings of association for machine translation in the Americas, pages 223-231. 